\title{
"SIGNS AND THE
}

BLINK OF AN EYE"

(SPEECH AND

PHENOMENA, CAPÍ-

TULO 5): LA CRÍTICA

DE DERRIDA AL

CONCEPTO

HUSSERLIANO DE

TIEMPO

Maite Zubiaurre*

\section{Contra la "metafísica de la presencia"}

Constituye el pensamiento derridiano uno de los más complejos y de más laboriosa lectura dentro de la historia de la filosofía y de la crítica literaria, disciplinas entre las que parece oscilar nuestro autor. Esta dificultad, sin embargo, es en cierta forma deliberada (Foucault, quien lo tuvo de alumno, lo tachaba de oscurantista) y producto, en todo caso, de la finalidad, tan peculiar y nueva en muchos aspectos, con que Derrida observa, no ya la realidad o las cosas, sino primordialmente los textos filosóficos. Su atención, como la de los pensadores afines al "textualismo", aparece, pues, desplazada del objeto al medio que la refleja.

* Centro de Lenguas, ITAM. 
MAITE ZUBIAURRE

Cree observar Derrida en la serie de textos representativos de la filosofía occidental (desde Platón hasta nuestros días) un mismo afán, una misma obsesión, una misma "creencia", en el sentido orteguiano, de la que el hombre de Occidente es inevitable prisionero: el "logocentrismo" o "fonocentrismo", dos términos que, aún sin ser exactamente sinónimos, constituyen reflejo de lo mismo y a veces, por tanto, funcionan como tales. Logocentrismo y fonocentrismo se definen de la siguiente manera: "(this) belief that the first and last things are the Logos, the Word, the divine Mind, the infinite understanding of God, an infinitely creative subjectivity and, closer to our time, the selfpresence of full self-consciousness".

Derrida, que contempla, pues, con profundo escepticismo la "ingenua" fe en un conocimiento humano, que rechaza enérgicamente toda actitud racionalista, no busca tampoco ser "comprendido" en el sentido ortodoxo de la palabra. Porque "entender" a Derrida significaría engrosar tan sólo el número de pensadores que pueblan la historia de la cultura occidental, esa cultura que es, para el pensador francés, pura "metafísica de la presencia".

Y si bien Derrida reconoce que no hay manera de escapar a esta 24 metafísica y que, por tanto, el uso de términos tradicionales se hace inevitable, intenta, sin embargo, no elevar ninguno de ellos a la categoría de concepto susceptible de definición o, al menos, de una sola definición. Hacerlo sería amoldarse irremisiblemente a lo que precisamente trata de combatir. Sería reconocer el valor absoluto de las cosas (considerar que a un significante le corresponde un solo significado, elevado a la categoría de primordial u originario), presuponerles un origen, soñar con él, como hacía Rousseau, y redondear inevitablemente el proceso, imaginando que el principio se verá clausurado con un final. A la "nostalgia" rousseauniana opone Derrida el resuelto "optimismo" de Nietzsche: "Turned toward the presence, lost or impossible, of the absent origin, (the) structuralist thematic of broken immediatiness

${ }^{1}$ Jacques Derrida, Of Grammatology, 1976, Baltimore and London, John Hopkins UP, p. XVIII. 
is thus the sad, negative, nostalgic, guilty, Rousseauist aspect of the thought of play of which the Nietzschean affirmation -the joyous affirmation of the play of the world of signs without fault, without truth, without origin, offered to an active interpretation-would be the other side." 2

Huye Derrida de lo acabado, de las leyes y normas que delimitan el espacio y dibujan los conceptos. Huye también de ese momento presuntamente "instantáneo" (punctual present) que es solamente presente, sin rastro de pasado, sin "huella" de futuro. Es la filosofía derridiana, en definitiva, un constante difuminar y borrar los límites, ya sean éstos espaciales, temporales o lingüísticos, y tal aspiración, claro está, ha de reflejarse también en la metodología.

Por eso, Derrida es antes que nada comentador minuciosísimo de las obras de los demás, por eso, el contenido de sus textos es, en proporción notable, "mero" comentario de otros textos. Quien comenta no asegura, no define, no asevera, tan sólo matiza, insinúa, describe. Y si concedemos a todo ello la dimensión política que ciertamente posee (conviene recordar que Derrida escribió sus tres obras fundamentales rondando el 68), habremos de reconocer que la definición, por su carácter rotundo, autoritario y ajeno a todo talante relativizador, es reflejo o metáfora oportuna de un sistema dictatorial y represivo.

Frente a la violencia de la definición, pues, el talante pacífico, "pacifista" y "demócrata" del comentario extenso y pacienzudo $\mathrm{y}$, como corolario de éste, curiosamente, breves definiciones también, pero de carácter "negativo". No nos dice Derrida lo que las cosas (y conceptos) son, sino lo que no son: "The pharmakon is neither the cure nor the poison, neither good nor evil, neither the inside nor the outside, neither speech nor writing; the supplement is neither a plus nor a minus, neither an outside, nor the complement of an inside (...); the gramme is neither a signifier nor a signified, neither a sign nor a thing, (...), etc." 3

2 Jacques Derrida, Writing and Difference, 1978, London, Routledge, p. 427.

3 Jaques Derrida, Positions, 1981, London, Atholne Press, p. 59. 
MAITE ZUBIAURRE

Qué duda cabe que esta negación "sintáctica", que con insistencia salpica el texto derridiano, refleja una actitud general apoyada en un "no" que trasciende la mera cuestión de estilo y que sistemáticamente combate el "sí" categórico (un sí al origen, a la presencia, al logos, etc.) de la cultura occidental. En cierto sentido, el "no" derridiano deja un hueco, al que el "sí" metafísico sirve de relleno y, por tanto, de "suplemento".

Harold Bloom, probablemente, $y$ a la vista de esa suerte de obstinada "pataleta" antimetafísica que parece aquejar a Derrida, no habría tardado en emitir su diagnóstico: una vez más, nos hallamos ante un hijo enzarzado en sangrienta lucha con su padre. La refriega, no obstante, es desigual. Derrida, que tan violentamente se revuelve contra el pensamiento metafísico, no puede, sin embargo, emplear otras armas que las que éste le brinda. Julia Kristeva, en una memorable entrevista al pensador francés, no vacila en subrayar tan flagrante paradoja: "How (...) could Derrida square his vigilant mistrust of metaphysical language with the fact that he had to work, of necessity, within that language, even while claiming to dismantle its whole conceptual structure?"4 Escuchemos la respuesta de Derrida: "If there is no possibility of breaking altogether with Western metaphysics, it is equally the case that every

26 text belonging, however rootedly, to that tradition bears within itself the disruptive potential of deconstructive reading." 5 "In every proposition or in every system of semiotic research (...) metaphysical presuppositions coexist with critical motifs. " ${ }^{6}$ También Derrida, pues, se ve obligado, inmerso como está en la cultura de Occidente, a convivir con la metafísica. Y tal convivencia, ya lo puntualizamos más arriba, no es pacífica ni conformista, sino casi siempre crítica y muchas veces deliberadamente corrosiva.

Refiriéndose a la "lectura crítica" afirma y no sólo comenta esta vez Derrida: "This moment of doubling commentary should no doubt have its place in a critical reading. To recognize and respect all its classical

${ }^{4}$ Christopher Norris, Deconstruction. Theory and Practice, 1982, London, p. 48.

\footnotetext{
${ }^{5}$ Ibidem.

${ }^{6}$ Jacques Derrida, Positions, op. cit., p. 36.
} 
exigencies is not easy and requires all the instruments of traditional criticism. Without this recognition and this respect, critical production would risk developing in any direction at all and authorize itself to say almost anything."7 Esta cita imaginamos que no será tan del gusto de los desconstruccionistas norteamericanos, para quienes, como puntualiza Norris, la desconstrucción es "a kind of hermeneutic free-for-all, a joyous release from all the rules and constraints of normative critical understanding". 8

El tono "ortodoxo" y tan extrañamente convencional, en un filósofo como Derrida, no debe, empero, llevarnos a engaño. El texto citado, de hecho, ofrece en cierta manera una visión distorsionada del pensamiento derridiano si no se le añade la frase que, a la vez que clausura el párrafo, lo critica ("desconstruye") y hasta invalida: "But this indispensable guardrail has always only protected, it has never opened a reading." El análisis tradicional -y casi vemos el gesto de triunfo de los desconstructores de Yale-, si bien es legítimo, no basta. Y no basta porque la lectura, lejos de conformarse con la pasiva actitud de "protegida", busca ella misma producir.

Basta un conocimiento superficial del pensamiento derridiano para saber identificar su principal objetivo, a saber: la demolición, no precipitada, sino cuidadosísima y calculada al milímetro, de esa prepotente "seguridad" con que la filosofía occidental acostumbra a fundamentar su discurso. Y el paso, no menos medido y estudiado, a un terreno que se encuentra más allá de la filosofía y en el que, inevitablemente, encuentra un lugar privilegiado el subconsciente, aun cuando Derrida tienda a desdeñar toda explicación "psicologista": "What I want to emphasize is simply that the passage beyond philosophy does not consist in turning the page of philosophy (which usually comes down to philosophizing badly) but in continuing to read philosophers in a certain way." Derrida no desea "salirse" de la filosofía, situarse fuera de ésta;

${ }^{7}$ Jacques Derrida, Of Grammatology, op. cit., p. 158.

${ }^{8}$ Ibidem, p. 139.

${ }^{9}$ Ibidem, p. 45. 
MAITE ZUBIAURRE

lo que busca, si acaso, es sumarse a las filas de esos pensadores "fronterizos" y de híbrida condición, de esos filósofos literatos, o escritores filósofos, al modo de Nietzsche, Adorno, Kirkegaard, Schopenhauer, Unamuno, el Hegel tardío. Representan todos ellos la lucha contra el racionalismo - ese racionalismo que llevó ya a Sócrates y a Platón a combatir la influencia contaminante del mito homérico-, la ruptura de una tradición purista y la instauración, en su lugar, de una actitud claramente romántica que, entre otras cosas, hizo posible el "descubrimiento" del subconsciente. Ello, además, explica no sólo esa afición de Derrida por el comentario, sino también el uso y aplicación de los conceptos filosóficos tradicionales.

No se trata, como vimos, de abandonar la filosofía, de "pasar la página", sino de leer a los filósofos "de una determinada manera". No se trata tampoco, añadiremos nosotros, de desdeñar los conceptos filosóficos, sino de tomarlos como de prestado y de añadirles otros nuevos, que no sean realmente conceptos, que no se revelen susceptibles de definición: tal es el caso de "differance", de "rastro", de "huella", de "suplemento" y de tantas otras aproximaciones derridianas.

El entero proceder escritural de Derrida es, sin duda, reflejo de su

28 pensamiento. Quien reniega de las verdades absolutas e insiste en la categoría deslizante y escurridiza de todo acontecer -quien cree que no hay presente sensu stricto, ni "presencia", sólo "rastro" de un "ahora" que nunca existió como tal, cuya esencia no reside en el contenido, sino en una forma que se repite, invariable- no puede, es claro, proponer nada que presente visos de originario, primordial, definitivo.

\section{Signos, instantes, rastros: Fenomenología y Desconstrucción opinan sobre el tiempo}

El capítulo 5 ("Signs and the Blink of an Eye") de Speech and Phenomena constituye, como los que le preceden y también los que le siguen, un extenso comentario a la fenomenología husserliana, aquejada, según Derrida, de ese "mal metafísico" que precisamente aspira a 
combatir. Husserl, en efecto, no sólo se revuelve contra el empirismo, el psicologismo y ese positivismo radical de las ciencias naturales que amenazaba con apoderarse del pensamiento filosófico, sino que rechaza de igual manera el idealismo kantiano, en un afán fenomenológico por regresar al objeto mismo, "colocando entre paréntesis" (la famosa epoché) todo lo que escapa a una experiencia o evidencia inmediatas. Sin embargo, Derrida pondrá constantemente en duda esa posibilidad de "inmediatez", esa posibilidad de percepción en un presente, y dada una presencia. Porque, para Husserl, la percepción necesita tanto de un sujeto como de un objeto, ambos enfrentados, el uno, pues, en presencia del otro. Derrida apuntará más tarde que la conciencia, en el sentido husserliano, es "autopresencia" (selfpresence), o "autoconciencia" (selfconciousness), puesto que se halla enfrentada a sí misma.

Afirma Derrida categóricamente en el capítulo 7 ("The Supplement of Origin") que "there never has been any perception" (una manera, pues, de asegurar que no hay presencia, que la "autoevidencia" no existe); en este otro capítulo que nos ocupa y que discute la noción husserliana del tiempo, demostrará el filósofo francés cómo el texto de su colega alemán se "desconstruye" a sí mismo, al afirmar que el presente, para posibilitar la experiencia, no puede reducirse a un mero "instante", sino que necesita además de una suerte de "pasado retenido" (retention) o de "presente prolongado" (extended present). Ello sería admitir que el "presente puro" no existe como tal y que, al no haber un presente "instantáneo", tampoco existe la posibilidad de una evidencia inmediata o pura.

Pero tal razonamiento, cargado de una lógica y precisión indiscutibles, aún lleva más allá y conecta con ese otro "punto flaco" en la filosofía husserliana, con esa polémica distinción entre "signo expresivo" y "signo indicativo", dentro de la que -y parece Derrida especialmente empeñado en hacerlo resaltar- este último representará siempre un papel desairado y claramente secundario. Un papel, por otra parte, que -nuestro filósofo es amigo a veces de llevar las cosas al extremohabrá de compartir con el lenguaje en general: "Husserl not only intends to exclude indication from "solitary mental life"; he will consider 


\section{MAITE ZUBIAURRE}

language in general (...) as a secondary event." ${ }^{10}$ Afirma Husserl que en el soliloquio (y una vez "liberado" el hombre de todo contacto exterior), que en "la vida solitaria del alma", recogiendo las propias palabras del filósofo, la expresión no necesita de signo indicativo, ya que "yo no me hago signos para entender lo que estoy pensando". " La expresión se presenta de forma "inmediata" y no necesita, por ello, de la "mediación" (y por tanto tampoco de la "mediatez") del signo indicativo. De la misma forma, la voz, según Husserl, posee la inmediatez que no posee la escritura, al hallarse ésta "diferida" por el signo, por el grafema; la escritura sería el "suplemento" de la voz, de la misma manera en que el signo indicativo funciona de "suplemento" de la expresión.

No obstante, la expresión en su estado "puro" (pure expression) y desembarazada de todo lastre indicador sólo se comprende si efectivamente la experiencia se manifestara en un instante verdaderamente "momentáneo", en un "ahora absoluto". De lo contrario, haría falta el signo indicativo para señalarnos, en el sentido de recordarnos o traernos a la memoria lo que ha ocurrido fuera de ese presente: "The force of this demonstration presupposes the instant as a point, the identity of experience instantaneously present to itself. Self-presence must be produced in the undivided unity of a temporal present so as to have nothing to reveal to itself by the agency of signs." 12

Es claro que si la "auto-presencia" (self-presence) no ocurre en un presente indiviso ( $\mathrm{y}$, de hecho, no cabe concebir una percepción instantánea), necesitaremos de signos indicativos para representarnos el pasado. Por lo tanto, si el presente -como afirma Husserl-contiene ya algo de pasado ("retención") e incluso de futuro, de anticipación ("pretensión"), el soliloquio no podrá prescindir de un componente indicativo (que nos "indique", ya sea rememorando o anticipando) y dejará, pues, de ser "expresión pura": "The fact that nonpresence and otherness

${ }^{10}$ Jacques Derrida, Speech and Phenomena, 1973, Evanston, Northwestern UP, p. 69.

${ }^{11}$ Julián Marías, Historia de la Filosofia, Revista de Occidente, p. 395.

${ }^{12}$ Jacques Derrida, Speech and Phenomena, op. cit., p. 60. 
are internal to presence strikes at the very root of the argument for the uselessness of signs in the self-relation." 13

Así, el signo indicativo, de alguna manera, "difiere" siempre el presente, lo aleja irremediablemente y siembra en nosotros esa ansia, esa nostalgia de algo que creímos que fue, que pensamos que estuvo presente, sin realmente haberlo estado nunca. Es ello probablemente la verdadera esencia del "rastro" o de la "huella", aproximación filosófica que apunta hacia lo deslizante, escurridizo e inaprehensible de toda realidad, hacia una verdadera falta de origen que Derrida opone al concepto husserliano de presencia o auto-evidencia (selfevidence) originarias; entre tanto sabemos, o al menos sospechamos, gracias a la labor desconstructora, que, por culpa de la infiltración contaminante del pasado, tal concepto ha tenido que sacrificar mucho de su firmeza y rotundez.

Sirva de ejemplo a esa "nostalgia" la curiosa paradoja (que recoge un poema de Thomas Hardy comentado por Hillis Miller) del hombre que, lamentándose de no haber prestado en su momento la necesaria atención a los seres que se cruzaron en su camino, pierde, mientras se conduele, ensimismado, la visión del paisaje que le rodea, vuelve a perder absurda e inevitablemente el presente. Se crea, pues, un juego constante de dilaciones susceptibles de ser "suplementadas" y que produce la engañosa impresión de un presente aprehensible. La presencia como tal (esa pura auto-presencia del yo) no es real sino ficticia, no es directamente perceptible o comprobable, sino mera metafísica, la "metafísica de la presencia".

Husserl distingue entre un pasado propiamente dicho, que necesita de reproducción, imaginación o representación que necesita de una memoria "secundaria", y ese pasado retenido, presentado y producido -ya no re-presentado o re-producido, ya no meramente imaginado sino genuinamente percibido-que, sujeto a una memoria "primaria" e inmediata, "goza de todos los privilegios del presente": "Husserl admittedly says that retention is still a perception." ${ }^{14} \mathrm{~A}$ la declaración que sigue ("...if we

${ }^{13}$ Ibidem, p. 66.

${ }^{14}$ Ibidem, p. 64. 
call perception the act in which all "origination" lies, which constitutes originarily, then primary remembrance is perception. For only in primary remembrance do we see what is post; only in it is the post constituted, i.e., not in a representative but in a presentative way") opone Derrida el siguiente comentario: "The difference between retention and reproduction, between primary and secondary memory, is not the radical difference Husserl wanted between perception and nonperception; it is rather a difference between two modifications of nonperception." 15

Es claro que nuestro pensador no admite ese valor de inmediatez y presencia que según Husserl anida en el pasado retenido, y que considera a éste tan poco susceptible de "percepción" como ese otro pretérito recuperable tan sólo mediante la memoria secundaria. Coincide con Husserl, eso sí, en que toda experiencia, por el solo hecho de serlo, necesita desarrollarse dentro de un lapso lógicamente más prolongado que un puro "guiño" instantáneo. Ya tuvimos ocasión de observar cómo reconoce a su vez la necesidad filosófica de ese "ahora". Sin embargo, el filósofo alemán, para justificar con la necesaria convicción la posibilidad de una evidencia absoluta (de una evidencia de una experiencia), y puesto que el simple Augenblick no basta (ya que no hay experiencia 32 sin continuidad), necesita en cierta forma "estirar" el presente y hacerlo extensivo, tanto a un pasado, el más próximo (retention) como a un futuro, el más inmediato (protention).

Pero semejante "estiramiento", según Derrida, tiene algo de forzado y por ello mismo delata cierto sentimiento de vacilación o de desasosiego en el propio Husserl: "The force with which he mantains that retention and protention belong to the sphere of the primordial(...) and the insistence with which he contrasts the absolute validity of primary memory with the relative validity of secondary memory, clearly indicate both his intent and his uneasiness." Esta inseguridad nace de querer combinar dos posturas irreconciliables:

a) The living now is constituted as the absolute perceptual source only in a state of continuity with retention taken as

${ }^{15}$ Ibidem, p. 65. 
nonperception. Fidelity to experience and to 'the things themselves' forbids that it be otherwise. b) The source of certitude in general is the primordial character of the living now; it is necessary therefore to keep retention in the sphere of primordial certitude and to shift the frontier between the primordial and the nonprimordial. The frontier must pass not between the pure present and the nonpresent, (...) but rather between two forms of the re-turn or res-titution of the present: re-tention and re-presentation. ${ }^{16}$

Lo cierto es que determinar el lugar exacto por el que ha de transcurrir la línea de separación entre pasado y presente escapa a una consideración filosófica y pasa, en cierta manera, a ser materia opinable. Sea como fuere, sirva esta cita de resumen de lo ya dicho. $Y$ añadamos que Derrida, por su parte, opone a esa "extensión" del presente una reducción absoluta de éste. No sólo rechaza la división husserliana entre retención y reproducción, entendida ésta como no-percepción y aquélla como percepción ("the difference between retention and reproduction (...) is rather a difference between two modifications of nonperception"), sino que considera el mismo presente -ese presente que, según su concepción, queda limitado a un estricto "ahora"- completamente ajeno a toda posibilidad de percepción.

Ello significa que para el filósofo francés el "ahora" -la presencia como source-point-, en cierta forma, no existe: "It is difficult to think of an origin without wanting to go back beyond it", nos dice Eagleton: ${ }^{17}$ "We must begin wherever we are and the thought of the trace (...) has already taught us that it is impossible to justify a point of departure absolutely." 18 Derrida se vuelve aún más explícito: "We thus come to posit presence - and, in particular, consciousness, the being-next-to-

${ }^{16}$ Ibidem, p. 66-7.

${ }^{17}$ Terry Eagleton, Literary Theory. An Introduction, 1983, Minneapolis, Minnesota UP.

${ }^{18}$ Jaques Derrida, Of Grammatology, op. cit., p. 62. 


\section{MAITE ZUBIAURRE}

itself of consciousness- no longer as the absolutely matrical form of being but as a 'determination' and an 'effect'. Presence is a determination and effect within a system which is no longer that of presence but that of differance." ${ }^{19} \mathrm{El}$ simple hecho, pues, de que la presencia sea siempre "efecto" de algo (efecto, quizás, de una ausencia) y no causa, le roba su valor de algo originario y primordial: "However far back we try to push (...) we discover that we must assume prior organization, prior differentiation. (...) We find only nonoriginary origins." 20

Lejos de constituir una entidad, un "contenido" real, la presencia es, a lo más, -y aparte, como vimos, de un concepto "imprescindible" dentro de la filosofia ("within philosophy there is no possible objection concerning this privilege of the present-now") -una nostalgia, una obsesión, una metafísica: la metafísica de la presencia. Ese "ahora", esa "autoevidencia", ese momento "original" y único sólo se admiten como sueño, como un anhelo más entre el abanico de nostalgias hasta cierto punto sinónimas (la nostalgia del origen, la nostalgia de Dios o del Logos, la nostalgia rousseauniana de la "Naturaleza inmaculada", la nostalgia también de la pureza y "transparencia" de la voz, etc.) que invaden al hombre occidental. El "ahora" se halla, como en la filosofía 34 husserliana, contagiado tanto de pasado como de futuro, y este pasado retenido o futuro anticipado en el ahora es, precisamente, el rastro.

El desacuerdo esencial entre ambos filósofos, empero, radica en que al afán de presencia y origen de Husserl opone Derrida ese otro afán, no menos obsesivo, por la "diferancia" (differance), por los momentos nunca originarios que se diluyen en un "juego de huellas" (play of traces), por el molde, la forma (y no contenido) ideal que se repite, inalterable, y que garantiza el regreso incesante del ahora. El paralelismo, por otra parte, entre esta forma ideal y el "significado ideal" (ideal meaning) en la filosofía husserliana nos lleva a pensar en un Derrida

${ }^{19}$ Jacques Derrida, Writing and Difference, op. cit., p. 47.

${ }^{20}$ Jonathan Culler, On Deconstruction. Theory and Deconstruction after Structuralism, 1982, Ithaca, Cornell UP, p. 96. 
inmerso en un idealismo metafísico que, aun apoyándose sobre premisas diferentes, se parece mucho al kantiano:

We should be able to say a priori that (the) common root (of retention and representation) -the possibility of re-petition in its most general form, that is, the constitution of a trace in the most universal sense-is a possibility which not only must inhabit the pure actuality of the now but must constitute it through the very moment of differance it introduces. Such a trace is (...) more "primordial" than what is phenomenologically primordial. For the ideality of the form of presence itself implies that it be infinitely repeatable, that its re-turn, as a return of the same, is necessary ad infinitum and is inscribed in presence itself. ${ }^{21}$

Nos hallamos, pues, ante un ahora que es siempre "rastro", que es también futuro, que es repetición constante de una forma siempre igual (el "ahora", como "ahora", será siempre lo mismo). Esa constante repetición de lo mismo es precisamente lo que garantiza la posibilidad de la experiencia: sin el rastro, sin la huella, no habría modo de experimentar el tiempo, ni de experimentar la presencia; por ello, se da la extraña paradoja de que, aun siendo la huella algo que en principio tendría que aparecer "después" del presente (un rastro es siempre algo que otra cosa deja tras de sí) es, sin embargo, anterior a éste. En ese sentido, cabría hablar de "huella original" (original trace) y cabría añadir, siguiendo con la misma paradoja, que es posible el rastro de algo que nunca ha existido. Husserl se opone a concepción tan "absurda", argumentando, en aparente buena lógica, que no puede memorizarse nada que no se haya experimentado con antelación: "It is certainly an absurdity to speak of a content of which we are "unconscious", one of which we are conscious only later (nachträglich)." 22 Hemos de aclarar, empero, que la fenomenología no contemplaba ese otro mundo,

${ }^{21}$ Jacques Derrida, Speech and Phenomena, op. cit., p. 67.

22 Ibidem, p. 63. 
MAITE ZUBIAURRE

ajeno todavía en buena parte al conocimiento humano, que llamamos el subconsciente. Y, sin embargo, por aquellos mismos años Freud estudiaba cómo una serie de procesos subconscientes eran muy capaces de determinar el posterior desarrollo de la vida de la consciencia.

\section{Derrida, filósofo metafísico, poeta romántico}

Los conceptos de origen y presencia, tan fundamentales en la crítica derridiana a la fenomenología, hemos visto que vienen acompañados de otros términos "satélite" de corte tradicional (tales como "percepción", "expresión" frente a "indicación", etc.) y más adelante, de una serie de pseudocategorías filosóficas, mucho menos ortodoxas y, hasta cierto punto, intercambiables: "huella", "rastro", "diferancia", "suplemento", etc. En el presente trabajo, que ha querido centrarse en la polémica derridiana sobre el concepto de tiempo en Husserl, no tienen cabida aclaraciones adicionales de esas otras categorías. No obstante, conviene recordar que la unidad de la obra derridiana se halla determinada precisamente por la sintomática frecuencia con que estos conceptos, tan difíciles de definir satisfactoriamente, salpican la obra del pensador francés. Son complicado retrato de sus obsesiones (u obsesión, puesto que, a la postre, todo se reduce a esa suerte de fobia a la por él bautizada "metafísica de la presencia"), a la vez que apoyo y guía del paciente lector. Éste, por otra parte, no tarda en percibir el peculiar idealismo que, seguramente muy a pesar de Derrida, trasciende de sus textos. Porque hay idealismo y metafísica, sin duda, en ese conceder valor trascendental y primordial al rastro, y hay, sobre todo, una gran dosis de romanticismo en el imperioso anhelo de extraer del olvido y poner sobre un pedestal lo que aparece como relegado y secundario. La huella, ya lo vimos, "is more 'primordial' than what is phenomenologically primordial". El signo indicativo es igualmente superior al signo expresivo. La escritura, esa gran marginada, lo es a la voz y al habla, etc. Por fin, los textos de segunda fila (como la obra rousseauniana que analiza en Of Grammatology), las notas de pie de 


\section{LA CRÍTICA DE DERRIDA}

página y hasta un simple trozo de papel que recoge una frase anodina -el paraguas olvidado de Nietzsche- merecen la más cuidadosa atención de Derrida. Hay romanticismo, insistimos, en ese "amor a la bagatela"; pero idealismo y romanticismo no son los únicos "ismos" que se traslucen de su pensamiento: el existencialismo, sin duda, completa y "suplementa", en el más puro sentido derridiano, la trinidad. 